\title{
Virtual MEG Helmet : Computer Simulation of an Approach to Neuromagnetic Field Sampling
}

\section{Medvedovsky, Mordekhay}

2016-03

Medvedovsky, M , Nenonen , J Koptelova, A, Butorina, A, Paetau, R, Makela , J P , Ahonen, A , Simola , J , Gazit , T \& Taulu , S 2016 , ' Virtual MEG Helmet : Computer Simulation of an Approach to Neuromagnetic Field Sampling ' , IEEE Journal of Biomedical and Health Informatics, vol. 20 , no. 2 , pp. 539-548 . https://doi.org/10.1109/JBHI.2015.2392785

http://hdl.handle.net/10138/161273

https://doi.org/10.1109/JBHI.2015.2392785

publishedVersion

Downloaded from Helda, University of Helsinki institutional repository.

This is an electronic reprint of the original article.

This reprint may differ from the original in pagination and typographic detail.

Please cite the original version. 


\title{
Virtual MEG Helmet: Computer Simulation of an Approach to Neuromagnetic Field Sampling
}

\author{
Mordekhay Medvedovsky, Jukka Nenonen, Alexandra Koptelova, Anna Butorina, Ritva Paetau, Jyrki P. Mäkelä, \\ Antti Ahonen, Juha Simola, Tomer Gazit, and Samu Taulu
}

\begin{abstract}
Head movements during an MEG recording are commonly considered an obstacle. In this computer simulation study, we introduce an approach, the virtual MEG helmet (VMH), which employs the head movements for data quality improvement. With a VMH, a denser MEG helmet is constructed by adding new sensors corresponding to different head positions. Based on the Shannon's theory of communication, we calculated the total information as a figure of merit for comparing the actual 306-sensor Elekta Neuromag helmet to several types of the VMH. As source models, we used simulated randomly distributed source current (RDSC), simulated auditory and somatosensory evoked fields. Using the RDSC model with the simulation of 360 recorded events, the total information (bits/sample) was 989 for the most informative single head position and up to 1272 for the VMH (addition of 28.6\%). Using simulated AEFs, the additional contribution of a VMH was $12.6 \%$ and using simulated SEF only $1.1 \%$. For the distributed and bilateral sources, a VMH can provide a more informative sampling of the neuromagnetic field during the same recording time than measuring the MEG from one head position. VMH can, in some situations, improve source localization of the neuromagnetic fields related to the normal and pathological brain activity. This should be investigated further employing real MEG recordings.
\end{abstract}

Index Terms-Internal magnetostatic multipole moments, magnetoencephalography, total information, virtual MEG helmet (VMH).

Manuscript received August 23, 2014; revised November 12, 2014; accepted January 8, 2015. Date of publication January 19, 2015; date of current version March 3, 2016. The work M. Medvedovsky was supported by Elekta Instrument AB/HI2838, Stockholm Sweden and by a Grant for project development by HUSLAB MLE82TK005, Helsinki Finland, 2007-2009, and the work of T. Gazit was supported by the European Union ACTIVE Grant FP7-ICT2009-270460. The Moscow MEG-Center was supported by The Ministry of Education and Science of Russian Federation (Grant RFMEF161914×0006). M. Medvedovsky and J. Nenonen contributed equally to this paper.

M. Medvedovsky is with the Brain Function Imaging Unit, Tel-Aviv Sourasky Medical Center, 64239 Tel-Aviv, Israel and also with BioMag Laboratory, HUS Medical Imaging Center, University of Helsinki and Helsinki University Hospital, 00029 Helsinki, Finland (e-mail: mamedvedovsky@ gmail.com).

J. Nenonen, A. Ahonen, and J. Simola are with the Elekta Oy, 00531 Helsinki, Finland (e-mail: jukka.nenonen@elekta.com; antti.ahonen9@gmail.com juha.simola@elekta.com).

A. Koptelova and A. Butorina are with the Center of Neurocognitive Research (MEG-center), Moscow State University of Psychology \& Education, Moscow 127051, Russia (e-mail: koptelova.am@gmail.com; armature@yandex.ru).

R. Paetau is with the Department of Pediatric Neurology and also with the Department of Clinical Neurophysiology, HUS Medical Imaging Centre, HUS/HUCH , FIN-00029 Helsinki, Finland (e-mail: ritva.paetau@hus.fi).

J. P. Mäkelä is with the BioMag Laboratory, HUS Medical Imaging Center, University of Helsinki and Helsinki University Hospital, FI-00029 Helsinki, Finland (e-mail: jyrki.makela@hus.fi).

T. Gazit is with the Brain Function Imaging Unit, Tel-Aviv Sourasky Medical Center, 64239 Tel-Aviv, Israel (e-mail: tomergazit@gmail.com).

$\mathrm{S}$. Taulu is with the Institute for Learning and Brain Sciences and Department of Physics, University of Washington, Seattle, WA 98195-58552 USA and also with Elekta Oy, FIN-00531 Helsinki, Finland (e-mail: staulu@uw.edu).

Digital Object Identifier 10.1109/JBHI.2015.2392785

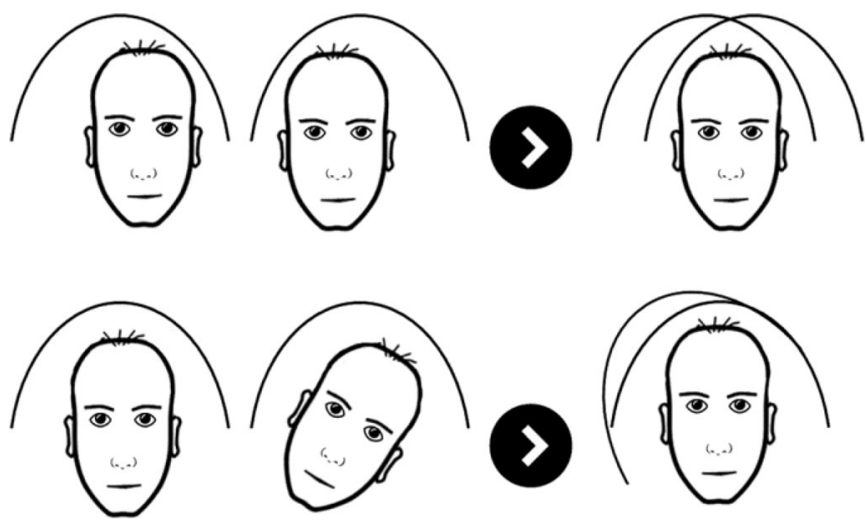

Fig. 1. Virtual MEG helmet construction. Upper row-translation. Lower row-rotation.

\section{INTRODUCTION}

B RAIN electrical activity is one of the most complex phenomena in human biological and medical research. To characterize this activity, the selected imaging technique should have as many independent information channels as possible. The modern MEG devices measure weak magnetic fields with hundreds of sensors. Nevertheless, the magnetic field sampling using even whole head MEG systems can be improved further. Nurminen et al. [1], [2] demonstrated in both simulations and experimental studies that the quality of data can be improved by supplementing, e.g., the 306-sensor MEG device (Elekta Neuromag, Elekta Oy, Finland), device with more sensors at different angles and at different layers. Adding such sensors, however, unavoidably increases device complexity and cost.

Alternatively, the number of sensors can be increased virtually by reconstructing an MEG helmet from different head positions and interpolating the recorded data. A change in head position within a static sensor array can be equivalently regarded as a change in sensor array position over a static head (see Fig. 1). If activity from the same sources is recorded in different head positions, this allows a virtual increase of the number of sensors by a factor of the number of head positions. We called this approach the virtual MEG helmet (VMH).

The influence of the MEG array sensor number (array density) on the source localization accuracy was studied by Vrba et al. [3]; it was demonstrated that equivalent current dipole (ECD) source localization does not improve further when more than 100-200 sensors are added to the array. When beamformer was used instead of ECD, addition of up to thousands sensors still improved the source localization accuracy. Notably, with VMH the increase in sensor number is expressed not only in higher 
density of sensor array, but also in higher variability in the sensor locations and directions: the VMH sensors are "placed" at different layers and at different angles (see Fig. 1), which potentially can enrich the neuromagnetic field sampling.

The disadvantage of the $\mathrm{VMH}$ is higher channel noise level compared with recording the same number of events in one head position. When MEG signals are measured in one head position, there are more events for averaging than when the signals from different head positions are used for the VMH construction. If $g$ is the number of events, then in a single head position, averaging of $g$ events reduces random noise by $\sqrt{g}$. However, for the VMH construction, signals recorded in $h$ different head positions are not averaged. If the events are equally distributed between head positions, the number of $\mathrm{VMH}$ averages is $g / h$ and the noise suppression is $\sqrt{g / h}$. Hence, comparing VMH and single head position recording, two oppositely directed tendencies can be observed.

1) The VMH possibly increases the number of independent MEG channels (increase of the forward model matrix rank).

2) The VMH is associated with $\sqrt{h}$ times less efficient noise suppression, where $h$ is the number of head positions and the recorded events are evenly distributed between recordings in different head positions.

The question addressed by this study is whether recording the same brain activity in different head positions and subsequent interpolation of the recorded data (VMH approach) can improve the data quality compared to recording the same number of events in one head position. In order to evaluate this question, we employed the total information calculation (Shannon's channel capacity [4]), assessing the merit of the different MEG sensor arrays [5], which was performed in two ways: 1) sensor level orthogonalization (SLO) of the lead fields [5] and 2) internal magnetostatic multipole moments (IMMM) signal-to-noise ratio (SNR). The IMMM SNR was calculated after modeling the magnetic field by a spherical harmonic vector basis, as implemented in the signal space separation (SSS) method [6].

The idea of virtual reconstruction of the brain magnetic field is not new. During the 1980s, MEG devices contained only one or a few channels, and the same brain activity was recorded several times, changing the position of the sensor relative to head. For example, Barth et al. [7] recorded several stereotypic epileptic interictal spikes in a patient with focal epilepsy by changing the position of one-sensor MEG device, and later, reported source localization of stereotypic epileptic seizures recorded in the same way [8]. Simultaneously recorded EEG was used to confirm the similarity of the brain activations for MEG signal interpolation. With the development of whole head MEG devices [9], the measurements were generally performed in one stable head position. In this paper, we are testing the hypothesis that changing the head position with respect to the whole head MEG sensor array, still can add information.

It is important to note that the spatial source (and signal) stability is essential for the applicability of the VMH. A VMH can be used with the spatiotemporally stable, especially externally triggered data, such as evoked responses. A VMH cannot be applied to spatiotemporally unstable data, which is often, but probably not always, the case with the spontaneous activity. It is clear that a VMH is not suitable for single event analysis.

A preliminary version of this study has been presented as an abstract [10].

\section{Methods}

\section{A. Total Information Calculated Using SLO of Lead Fields}

According to Shannon's theory of communication, if the signal $b(t)$ and the noise noise $(t)$ are normally distributed and independent, the total information $\left(\mathrm{Inf}_{\mathrm{tot}}\right)$ provided by the single noisy channel can be described as:

$$
\operatorname{Inf}_{\text {tot }}=1 / 2 \log _{2}(P+1)
$$

where $P$ is the power SNR of the single channel. Then, the total information provided by the system of $m$ independent channels can be represented as

$$
\operatorname{Inf}_{\text {tot }}=1 / 2 \sum_{n=1}^{m} \log _{2}\left(P_{n}+1\right)
$$

where $P_{\mathrm{n}}$ is the power SNR of each independent channel. Thereby, an increase of the number of independent channels will increase the total information, whereas increase of noise will decrease it. The total information of MEG sensor arrays was first suggested by Kemppainen and Ilmoniemi [5], and later studied by Nenonen et al. [11], [12]. All these studies used the assumption that the source current and the noise are randomly distributed. This is actually a simplification, because in the reality neither the source current nor noise is distributed completely randomly. In this study, we use the term "randomly distributed source current" (RDSC) for this type of source model.

The source was assumed to be distributed throughout the spherical brain model with a radius of $8 \mathrm{~cm}$.

In a multichannel array, the coupling of channel $n$ is quantified in terms of the lead field $L_{n}$, according to

$$
b_{n}=\int L_{n}\left(r^{\prime}\right) \cdot j_{p}\left(r^{\prime}\right) d v^{\prime}
$$

where $r$ ' is a vector, which indicates location of the neural source, the center of the coordinate system is located at the center of spherical conductor; $j_{\mathrm{p}}\left(r^{\prime}\right)$ is the primary current density vector at $r^{\prime}$; and $v^{\prime}$ is a spherical conductor volume.

As in previous works dealing with total information of MEG systems (see [5], [11], and [12]), in this study, we assume that no a priori information of the sources exists, and that the primary currents have a Gaussian distribution, $j_{\mathrm{p}} \sim N\left(0, s_{\mathrm{p}}{ }^{2}\right)$. In addition, the noise is also assumed Gaussian, noise ${ }_{\mathrm{n}} \sim N(0$, $\sigma_{\mathrm{n}}{ }^{2}$ ). Thus, the power SNR for channel $n$ is

$$
P_{n}=\left\|L_{n}\right\|^{2} s^{2} / \sigma_{n}^{2} .
$$

As stated, the total information, $\operatorname{Inf}_{\text {tot }}$, can be evaluated as a sum of $\log _{2}\left(P_{\mathrm{n}}+1\right)$ over independent channels. Because the lead fields of the sensors are overlapping, sensor measurements are dependant, and hence, the data must be orthogonalized. For this purpose, a lead field product matrix (gram matrix) is 
constructed according to

$$
G_{j k}=\int L_{j}\left(r^{\prime}\right) \cdot L_{k}\left(r^{\prime}\right) d v^{\prime} .
$$

Next, we apply singular value decomposition of $G$ according to $G=U S U^{T}, S=\operatorname{diag}\left(\lambda_{1}, \lambda_{2}, \ldots \lambda_{m}\right)$, where the columns of matrix $U$ are the eigenvectors and $\lambda_{n}$ are the eigenvalues of $G$. The orthogonalized lead fields become $L_{n}{ }^{\prime}=U^{T} L_{n}$ and the orthogonalized SNR becomes

$$
P_{n}^{\prime}=\lambda_{n} s^{2} / \sum_{j}\left(U_{n j} \sigma_{j}\right)^{2} .
$$

The total information of the $m$-channel magnetometer per sample is thus

$$
\operatorname{Inf}_{\text {tot }}=1 / 2 \sum_{n=1}^{m} \log _{2}\left(P_{n}^{\prime}+1\right) .
$$

In the simulations, the nonaveraged root mean square (RMS) noise level was set to $30 \mathrm{fT} / \sqrt{\mathrm{Hz}}$ for magnetometers and to 10 $\mathrm{fT} / \mathrm{cm} / \sqrt{\mathrm{Hz}}$ for planar gradiometers-representing the situation in which the human head is inside the MEG helmet and the noise level is dominated by brain background activity.

In our simulations, the standard deviation of primary source current density, $j_{\mathrm{p}}$ was defined as $0.6 \mu \mathrm{A} / \mathrm{m}^{2}$ so that the magnetic field RMS value of $100 \mathrm{fT} / \sqrt{\mathrm{Hz}}$ can be measured at $20 \mathrm{~mm}$ outside the scalp [9].

\section{B. Total Information Calculated Using IMMM SNR}

The SSS method transforms MEG data into sets of multipole moments, which correspond to different vector spherical harmonic functions [6]. This method, together with its temporal extension (spatio-temporal signal space separation - tSSS [13]), is employed in interference suppression and in head movement compensation [14], [15]. Typically, the noise-based truncation of the harmonic bases leads to 80 internal and 15 external basis functions. SSS transforms the sensor-level signals of interest and part of the random noise into internal multipole moments and their SNR values depend on both transformed noise and transformed signal of interest. With $h$ head positions, there are $h$ times less events for averaging, and therefore, the VMH channel noise level is $\sqrt{h}$ times higher than with one head position. The overall amplitude of transformed noise depends linearly on the noise amplitude of the MEG channels of a given sensor array. However, SSS transformation of both signal and noise is influenced by the sensor array geometry, and therefore, multipole moments SNR can be different in VMH when compared to real MEG array. Because SSS transformation creates an orthogonal basis, $P_{\mathrm{n}}$ in (2) can be also the power SNR of IMMM, therefore, it can also serve to total information computing. The calculation of total information using IMMM SNR was described in [12].

The SSS transformation was performed for both reference head position in the 306-sensor helmet (Stand_1) and for three types of the VMH: VMH_comb2_3, VMH_comb3, and VMH7_2 (see Fig. 5). The IMMMs were calculated separately for signal of interest and for random noise. Then, SNR of IMMMs was defined as the ratio of signal multipole moments and noise multipole moments for every internal spherical harmonic. The total information was calculated using (2), where $P_{\mathrm{n}}$ is IMMM SNR. We compared the total information calculated using SLO and IMMM SNR approaches (see Table II). In a first set of simulations (see IIand Fig. 5), the signal of interest was defined as RDSC. Here, we calculated a random overall current distribution in the brain, followed by the calculation of the associated internal multipole moments and transformed a sensor-level noise matrix into the internal multipole moments. The magnetometer noise of nonaveraged data was set on 30 $\mathrm{fT} / \sqrt{\mathrm{Hz}}$ and planar gradiometer noise - on $10 \mathrm{fT} / \mathrm{cm} / \sqrt{\mathrm{Hz}}$ (the same as in total information computing). The absolute values of the source current and sensor noise are irrelevant as we are studying the relative differences of the SNRs between the 306channel and VMH constructions. The channel noise was defined for 306-sensor helmet $\sqrt{h}$ times lower than for the VMH, where $h$ is number of head positions.

In addition to RDSC, we simulated the auditory evoked fields (AEFs) and the somatosensory evoked field (SEF). We assumed that the sources of AEFs can be represented by two equivalent current dipoles (ECDs) located bilaterally, and the source of SEF by a single unilateral ECD. The locations and orientations of the ECDs were defined by fitting the dipoles using the measured data published by Nenonen et al., 2010 [16]. The location of SEF source was $x=-34.9, y=5.1$, and $z=104.4$. The locations of AEF sources (head coordinates, $\mathrm{mm}$ ) were: $x=-57.0, y=2.5, z=64.0$, and $x=54.0$, $y=10.0, z=62.0$. The source amplitudes were set at $50 \mathrm{nAm}$. The noise was set at $\sqrt{h} 5 \mathrm{fT} / \mathrm{cm} / \sqrt{\mathrm{Hz}}$ for planar gradiometers and $\sqrt{h} 20 \mathrm{fT} / \sqrt{\mathrm{Hz}}$ for magnetometers, where $h$ is the number of head positions. In order to calculate total information for AEF and SEF, we employed the SSS method and calculated power SNR of IMMM as in [12]. The results of the AEF and SEF simulations are presented in two last columns of Table I and in Fig. 2.

\section{VMH Construction}

Sensor locations were introduced to the model according to the Cartesian coordinates of the 306 MEG sensor (102 magnetometers and 204 planar gradiometers) positions and directions of the Elekta Neuromag MEG device. The sensor position was defined as a vector from the device coordinate system origin to the geometrical center of the sensor. The sensor orientation was defined by three unit vectors corresponding to Cartesian $x-y$-, and $z$-axes of the sensor coordinate system translated into the device coordinate system origin.

In the device coordinate system, the $x$-axis was directed to the right, $y$-axis to forward, and $z$-axis to upward. The virtual arrays were constructed by MEG array displacements in the coordinate system of the head. The head coordinate system had the origin in the center of the spherical brain model, which was located at $x=0, y=0, z=-40 \mathrm{~mm}$ point of the device coordinate system in the reference head position. The $x$ -,$y$-, $z$-axes of the head and device coordinate systems had the same directions in the reference head position. In such position, an adult-size head has no space to move backward or upward; this was taken into account in constructing the virtual arrays. 
TABLE I

Standard ANd Virtual MEG SENSOr ARrays ANd Head Positions

\begin{tabular}{|c|c|c|c|c|c|c|c|c|c|c|c|}
\hline \multirow{4}{*}{$\begin{array}{l}\text { Array } \\
\text { index }\end{array}$} & \multirow{4}{*}{$\begin{array}{l}\text { Array (or head } \\
\text { position) name }\end{array}$} & \multirow{4}{*}{$\begin{array}{l}\text { Head positions (translations, } \\
\mathrm{mm} \text {; rotations, degrees) }\end{array}$} & \multirow{4}{*}{$\begin{array}{l}\text { Number of } \\
306 \text { sensor } \\
\text { array } \\
\text { duplications }\end{array}$} & \multicolumn{6}{|c|}{ RDSC } & \multirow{3}{*}{\multicolumn{2}{|c|}{\begin{tabular}{c|c} 
AEF & SEF \\
Total \\
information, \\
bit/sample
\end{tabular}}} \\
\hline & & & & \multicolumn{3}{|c|}{$\begin{array}{l}\text { RMS of magnetometer noise, } \\
\mathrm{fT} / \sqrt{\mathrm{Hz}}\end{array}$} & \multirow{2}{*}{\multicolumn{3}{|c|}{$\begin{array}{l}\text { Total information, bit/sample } \\
\text { ents: }\end{array}$}} & & \\
\hline & & & & \multicolumn{3}{|c|}{ Number of events: } & & & & & \\
\hline & & & & 360 & 720 & 1440 & 360 & 720 & 1440 & & \\
\hline 1 & Stand_1 & Ref & 1 & 1.581 & 1.118 & 0.791 & 857 & 973 & 1094 & 360 & 317 \\
\hline 2 & Stand_2 & Xrot-30 & 1 & 1.581 & 1.118 & 0.791 & 857 & 973 & 1094 & 316 & 311 \\
\hline 3 & Stand_3 & Yrot-30 & 1 & 1.581 & 1.118 & 0.791 & 857 & 973 & 1094 & 324 & 335 \\
\hline 4 & Stand_4 & Zrot-30 & 1 & 1.581 & 1.118 & 0.791 & 857 & 973 & 1094 & 307 & 363 \\
\hline 5 & Stand_5 & Xtrans-10 & 1 & 1.581 & 1.118 & 0.791 & 877 & 988 & 1103 & 246 & 436 \\
\hline 6 & Stand_6 & Ytrans +10 & 1 & 1.581 & 1.118 & 0.791 & 774 & 888 & 1008 & 300 & 331 \\
\hline 7 & Stand_7 & Ztrans-10 & 1 & 1.581 & 1.118 & 0.791 & 742 & 849 & 962 & 276 & 398 \\
\hline 8 & Stand_8 & $\begin{array}{l}\text { Combined movement: } \\
\{\text { xtrans }+10 ; \text { xrot- } 30 \\
\text { yrot-30; zrot }-30\}\end{array}$ & 1 & 1.581 & 1.118 & 0.791 & 989 & 1103 & 1221 & 258 & 273 \\
\hline 9 & Stand_9 & $\begin{array}{l}\text { Combined movement: } \\
\{\text { xtrans }-10 ; \text { xrot }+30 ; \\
\text { yrot }+30 ; \text { zrot }+30\}\end{array}$ & 1 & 1.581 & 1.118 & 0.791 & 989 & 1103 & 1221 & 357 & 437 \\
\hline 10 & VMH2_1 & Ref \& zrot-15 & 2 & 2.236 & 1.581 & 1.118 & 920 & 1056 & 1202 & 259 & 307 \\
\hline 11 & VMH2_2 & Ref \& zrot-30 & 2 & 2.236 & 1.581 & 1.118 & 940 & 1079 & 1228 & 292 & 329 \\
\hline 12 & VMH3_1 & Ref \& zrot-15 \& zrot-30 & 3 & 2.739 & 1.936 & 1.369 & 950 & 1093 & 1248 & 314 & 408 \\
\hline 13 & VMH2_3 & Ref \& xtrans-10 & 2 & 2.236 & 1.581 & 1.118 & 965 & 1102 & 1248 & 302 & 417 \\
\hline 14 & VMH2_4 & Xtrans $-10 \&$ xtrans +10 & 2 & 2.236 & 1.581 & 1.118 & 1052 & 1191 & 1340 & 263 & 398 \\
\hline 15 & VMH3_2 & Ref \& xtrans- $10 \&$ xtrans +10 & 3 & 2.739 & 1.936 & 1.369 & 1054 & 1203 & 1361 & 325 & 385 \\
\hline 16 & VMH3_3 & Ref \& xtrans-10 \& ytrans +10 & 3 & 2.739 & 1.936 & 1.369 & 965 & 1108 & 1263 & 318 & 320 \\
\hline 17 & VMH4_1 & $\begin{array}{l}\text { Ref \& xtrans-10 \& ytrans }+10 \& \\
\text { ztrans-10 }\end{array}$ & 4 & 3.162 & 2.236 & 1.581 & 954 & 1099 & 1256 & 280 & 442 \\
\hline 18 & VMH7_1 & $\begin{array}{l}\text { Ref \& xtrans-10 \& ytrans }+10 \& \\
\text { ztrans-10 \& } \\
\text { xrot-15 \& yrot-15 \& zrot-15 }\end{array}$ & 7 & 4.201 & 2.970 & 2.095 & 986 & 1140 & 1310 & 307 & 308 \\
\hline 19 & VMH7_2 & $\begin{array}{l}\text { Ref \& xtrans }-10 \& \text { xtrans }+10 \& \\
\text { ytans }+10 \& \text { xrot- } 30 \& \text { yrot-30 } \\
\& \text { zrot-30 }\end{array}$ & 7 & 4.201 & 2.970 & 2.095 & 1092 & 1260 & 1445 & 286 & 373 \\
\hline 20 & VMH_comb2_1 & $\begin{array}{l}\text { Ref \& }\{\text { xtrans- } 10 ; \text { ytrans }+10 ; \\
\text { ztrans-10; xrot-15; xrot-15; } \\
\text { zrot-15\} }\end{array}$ & 2 & 2.236 & 1.581 & 1.118 & 1071 & 1218 & 1375 & 288 & 415 \\
\hline 21 & VMH_comb2_2 & $\begin{array}{l}\text { Ref \& }\{\text { xtrans- } 10 ; \text { xtrans }+10 ; \\
\text { ytrans }+10 ; \text { xrot }-30 ; \text { xrot- } 30 ; \\
\text { zrot-30\} }\end{array}$ & 2 & 2.236 & 1.581 & 1.118 & 1193 & 1350 & 1517 & 356 & 364 \\
\hline 22 & VMH_comb2_3 & $\begin{array}{l}\{\text { Xtrans }+10 ; \text { xrot }+15 ; \operatorname{xrot}+15 \\
\text { zrot }+15\} \&\{\text { xtrans- } 10 ; \text { xrot- } 15 \\
\text { yrot- } 15 ; \text { zrot- } 15\}\end{array}$ & 2 & 2.236 & 1.581 & 1.118 & 1256 & 1414 & 1580 & 336 & 419 \\
\hline 23 & VMH_comb3 & $\begin{array}{l}\text { Ref \& }\{\text { xtrans }+10 ; \text { xrot }+15 ; \\
\text { yrot }+15 ; \text { zrot }+15\} \&\{\text { xtrans- } \\
10 ; \text { xrot- } 15 ; \text { yrot- } 15 ; \text { zrot- }-15\}\end{array}$ & 3 & 2.739 & 1.936 & 1.369 & 1272 & 1448 & 1636 & 406 & 379 \\
\hline
\end{tabular}

Ref — reference head position; $x$ trans — translation along axis $x$; ytrans — translation along axis $y$; ztrans — translation along axis $z$; $x$ rot — rotation around axis $x$, yrot — rotation around axis $y$; zrot — rotation around axis $z$. RDSC — randomly distributed source current. AEF — auditory evoked fields. SEF — somatosensory evoked fields. The direction and size of the head displacement is indicated by the number after the displacement name; for example, " $x$ rot- 30 " means rotation minus 30 degrees around axis $x$, or " $y$ trans +10 " means translation plus $10 \mathrm{~mm}$ along axis $y$. Different head positions are separated by $\&$, and the notation for different aspects of one combined head displacement is $\{\ldots\}$; for example: $\{x$ trans $+10 ; x$ rot-30; yrot-30; zrot -30$\}$. The head displacements are expressed as displacement of the head in terms of device coordinate system, which have opposite direction relative to sensor array virtual displacements (in a process of VMH construction. The name Stand_ means head position in the standard MEG array, and Virt ... means virtual MEG array. RMS — root mean square. The RMS of magnetometer noise is calculated according to 360, 720 , or 1440 events.

Fourteen different virtual sensor arrays were constructed with sensor numbers from $306 \times 2$ to $306 \times 7$ (see Table I: arrays 519). In addition, we simulated nine head positions (one reference and eight displaced ones) using the standard 306 sensor array alone (see Table I: arrays 1-9). The head displacements included 10-mm translations in all directions except for backward and upward (because of physical constraints set by the helmet) and rotations to $15-30^{\circ}$ around all axes. Translation of the helmet 


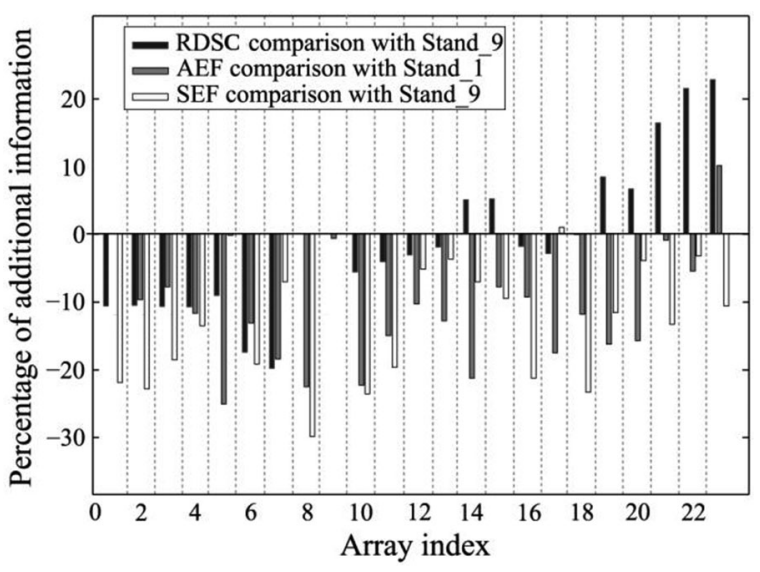

Fig. 2. Percent of additional information (PAI) computed for head positions indexed from 1 to 9 and for virtual arrays indexed from 10 to 23, compared with Stand_1 and with Stand_9. The description of virtual arrays and head positions is detailed in Table I according to array index ( $x$-axis of the Fig. 2 corresponds to the first column of the Table I). In the case of RDSC, the results presented in this figure were calculated based on situation with 360 recorded events.

origin was done first, and rotations in order z-rotation, $y$-rotation, $x$-rotation were combined into a single rotation matrix. Some of simulated head displacements (translations downward and forward) were in the range of the spontaneous head movements during MEG recording reported by Wehner et al. However, translations in the $x$-axis (sidewise) exceeded the range reported in [17]: $10 \mathrm{~mm}$ to one side in our study and $3.7 \mathrm{~mm}$ standard deviation (maximum about $5 \mathrm{~mm}$ to one side) in the study of Wehner et al.

The names,indices, and features of different head positions in both the standard array and in virtual arrays are presented in Table I. For a single head position in the standard MEG array, we used names Stand_1 to 9 (standard MEG array). Stand_1 corresponds to reference head position and Stand_2 to 9 correspond to displaced head positions in a standard MEG array. For different types of virtual arrays, constructed on the basis of more than one head position, we used the name VMH.

For construction of the different VMHs, we simulated both simple and combined head displacements. Simple head displacement was either translation along one axis or rotation around one axis. Combined head displacement was a head displacement, which included the combination of more than one simple head displacements (for example: combination of $x$-translation and $z$-rotation) in one head position.

\section{Number of Recorded Events Used for Randomly Distributed Source Current Simulation}

For randomly distributed source current simulation, we considered three situations of MEG recording with different number of events: 1) 360 events, 2) 720 events, 3) 1440 events.

The magnetometer noise of averaged data was calculated for the standard array and the VMH as

$$
\text { Noise }_{306}=30 \frac{\mathrm{fT}}{\sqrt{\mathrm{Hz}}} / \sqrt{\text { number of events }}
$$

$$
\text { Noise }_{\mathrm{vmh}}=30 \frac{\mathrm{fT}}{\sqrt{\mathrm{Hz}}} / \sqrt{\text { number of events } / h}
$$

where Noise $_{306}$ is the magnetometer noise level for one head position measurement in the 306-sensor MEG helmet and Noise $_{\mathrm{vmh}}$ the corresponding noise level for $\mathrm{VMH}$, and $h$ is the number of head positions.

The planar gradiometer noise was calculated employing the same formulae but using $10 \mathrm{fT} / \mathrm{cm} / \sqrt{\mathrm{Hz}}$ instead of $30 \mathrm{fT} / \sqrt{\mathrm{Hz}}$.

\section{E. Computing the Percentage of Additional Information (PAI)}

PAI of array A versus array B was defined as

$$
\mathrm{PAI}=\left(\left[\operatorname{Inf}_{\mathrm{tot} A} / \operatorname{Inf}_{\operatorname{tot} B}\right]-1\right) 100 \%
$$

where $\operatorname{Inf}_{\text {tot } A}$ is the total information obtained using array $\mathrm{A}$ and $\operatorname{Inf}_{\text {tot } B}$ using array $B$.

PAI was calculated for different head positions and different types of VMH versus reference head position in a standard helmet_-Stand_1 and versus displaced head position in standard helmet_-Stand_5 (see Fig. 2). The noise was estimated to correspond to $3 \mathrm{~h}$ recording with 360 events. With a standard 306-sensor helmet, all events were averaged together as in one head position, whereas with VMH events recorded in different head positions are averaged separately.

The dependence of PAI on number of events was studied (see Fig. 3) in one displaced head position in standard helmet [Stand_5-Fig. 3 (a)] and two types of VMH [VMH_comb3Fig. 3 (b) and VMH7_2-Fig. 3 (c)] compared to the reference head position in standard helmet (Stand_1). The calculations were performed for three clinical situations with 360, 720, and 1440 events.

Thereafter, we calculated total information provided by three types of VMH: VMH_comb2_3, VMH_comb3, and VMH7_2 and by the reference head position in a standard helmet as a function of magnetometer noise with RMS from 0.1 to $20 \mathrm{fT} / \sqrt{H z}$ (see Fig. 4). In this calculation, the planar gradiometer noise was corrected proportionally to changes in magnetometer noise.

\section{RESULTS}

\section{A. Essential Findings (see Fig. 2)}

1) Randomly Distributed Source: With 360 events (e.g., epileptic spikes), the total information (bits/sample) was 989 for the most informative head position in the standard helmet and up to 1272 for VMH (additional 28.6\%); with 720 events1103 for the most informative single head position in standard helmet and up to 1448 for VMH (additional 31.3\%); and with 1440 events - 1221 for the most informative single head position in standard helmet and 1636 for VMH (additional 34.0\%).

2) Simulated AEFs: The total information provided by the most informative head position in standard array was 360 bit/sample. By the most informative VMH, it was 406 bit/sample (additional 12.8\%).

3) Simulated SEF: The total information provided by the most informative single head position in standard array was 437 bit/sample. By the most informative VMH, it was 442 bit/sample (additional 1.1\%) 


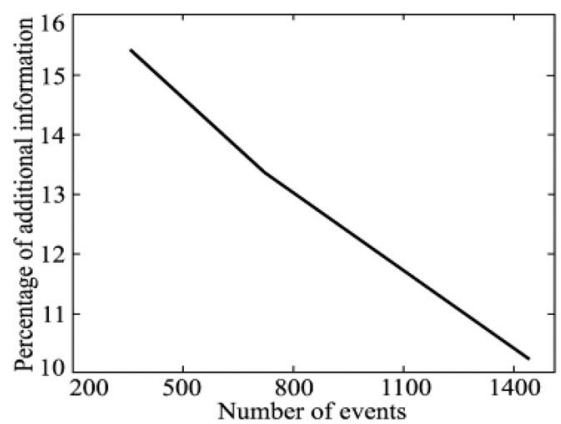

(a)

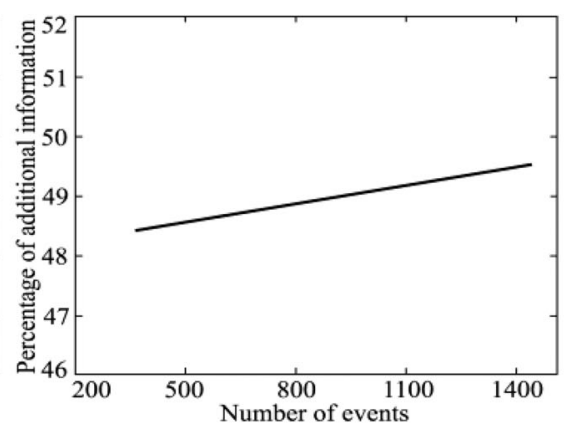

(b)

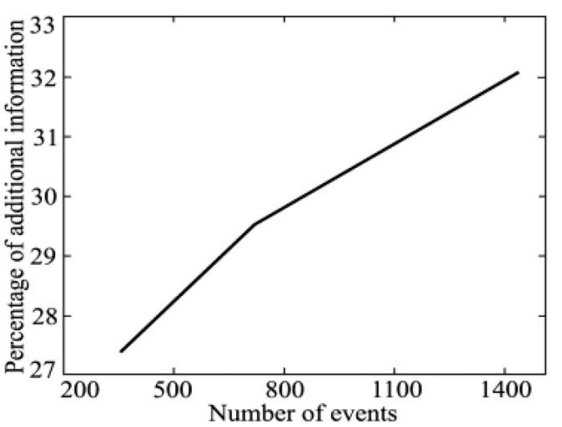

(c)

Fig. 3. Influence of numbers of events on the additional total information contribution by: (a) Stand_8, (b) VMH_comb3, and (c) VMH7_2 compared to Stand_1.

\section{B. Total Information: SLO With RDSC (see Table I and Fig. 2)}

1) Displaced Head Positions in the Standard MEG Array Compared to Stand_l (see Table I Arrays 2-9, and Fig. 2): Translation along $x$-axis (sidewise shift) to $10 \mathrm{~mm}$ (see Table I array 5) increased slightly the total information compared with Stand_1; the PAI was $2.3 \%$ in the situation with 360 events. $Y$ translation $10 \mathrm{~mm}$ forward (see Table I array 6) and $z$-translation $10 \mathrm{~mm}$ downward (see Table I array 7) were associated with total information reduction; with 360 events, PAI was $-9.7 \%$ and $-13.4 \%$, respectively. The single head positions in the standard MEG array that provided the highest PAI (15.4\%) were combined displacements: $x$-translation to $+10 \mathrm{~mm}$ and $x$-, $y$-, and $z$-rotations to $-30^{\circ}$ (see Table I array 8 [Stand_8]); and $x$-translation to $-10 \mathrm{~mm}$ and $x-, y$-, and $z$-rotations to $+30^{\circ}$ (see Table I array 9 [Stand_9]). Stand_8 and Stand_9 had the same values of total information with RDSC.

2) VMH (see Table I arrays 10-23 and Fig. 2): VMH versus the most informative single head position (Stand_8 or Stand_9). Majority of VMHs based on simple head displacements provided less total information than Stand_9 (negative PAI). Only two categories of VMHs had positive PAI compared with Stand_8. One was VMH with simple head displacements to opposite directions along $x$-axis (20-mm distance between head positions): VMH2_4 (see Table I array 10), VMH3_2 (see Table I array 11), and VMH7_2 (see Table I array 15). Another category was VMHs with combined head displacements (see Table I arrays 20-23); all had a positive PAI

3) PAI as a Function of Number of Events (see Fig. 3): Stand_8 demonstrated a decrease of PAI when number of events increased (and therefore, noise decreased) from $15.4 \%$ with 360 events to $11.6 \%$ with 1440 events [difference of $-3.8 \%$, Fig. 3 (a)]. The same test for VMH_comb3 revealed PAI increase from $48.4 \%$ with 360 events to $49.5 \%$ with 1440 events [difference of 1.1\%, Fig. 3(b)]; and for VMH7_2—increase from 27.4\% with 360 events to $32.1 \%$ with 1440 events [difference of $4.7 \%$, Fig. 3 (c)].

4) Total Information as a Function of Magnetometer Noise (see Fig. 4): With increase in noise, the total information decreased, as can be predicted based on (1) and (2), which is due to decrease of $P$. The arrays with higher number of sensors provided more total information with the same level of noise, which is due to increase of $m$ in (2) and (7).

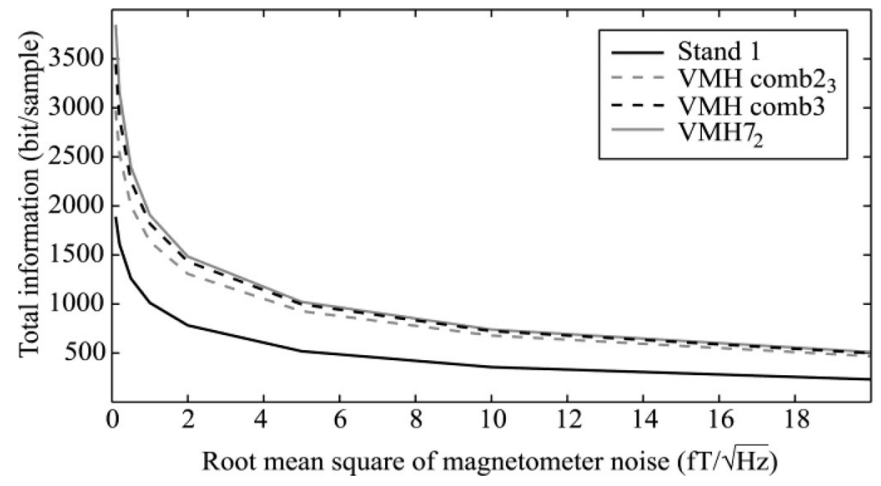

Fig. 4. Total information provided by Stand_1 and by three types of VMH (VMH_comb2_3, VMH_comb3, VMH7_2) as a function of root mean square of the magnetometer noise. The planar gradiometer noise in not shown, but it was corrected in proportion to the change of magnetometer noise.

TABLE II

Total Information: InTERnal Magnetostatic Multipole Moments VERSUS SLO

\begin{tabular}{lcccc}
\hline \hline & Stand_1 & VMH7_2 & VMH_comb2_3 & VMH_comb3 \\
\hline TIIMMM & 831 & 980 & 1190 & 1156 \\
TISLO & 857 & 1092 & 1256 & 1272 \\
PAIIMMM & & $17.9 \%$ & $43.2 \%$ & $34.9 \%$ \\
PAISLO & & $27.4 \%$ & $46.5 \%$ & $48.4 \%$ \\
\hline \hline
\end{tabular}

TIIMMM denotes Total Information calculated using IMMM. TISLO denotes Total Information calculated using SLO. PAIIMMM denotes PAI calculated using IMMM and PAISLO denotes PAI calculated using SLO

\section{Total Information: IMMM SNR With RDSC (see Table II and Fig. 5)}

The SNR was higher with all three types of VMH: VMH_comb3 and VMH7_2 as compared to reference head position in the standard 306-sensor helmet (Stand_1) for all 80 IMMMs (see Fig. 5, Table II). VMH7_2 created by seven simple head displacements had lower IMMM SNR than VMH types created by two or three combined movements (VMH-_comb2_3 and VMH_comb3). The total information computed based on the IMMM SNR calculations using the RDSC model was also higher with VMHs than with Stand_1 and was higher with VMHs created by combined displacements than with the VMH 


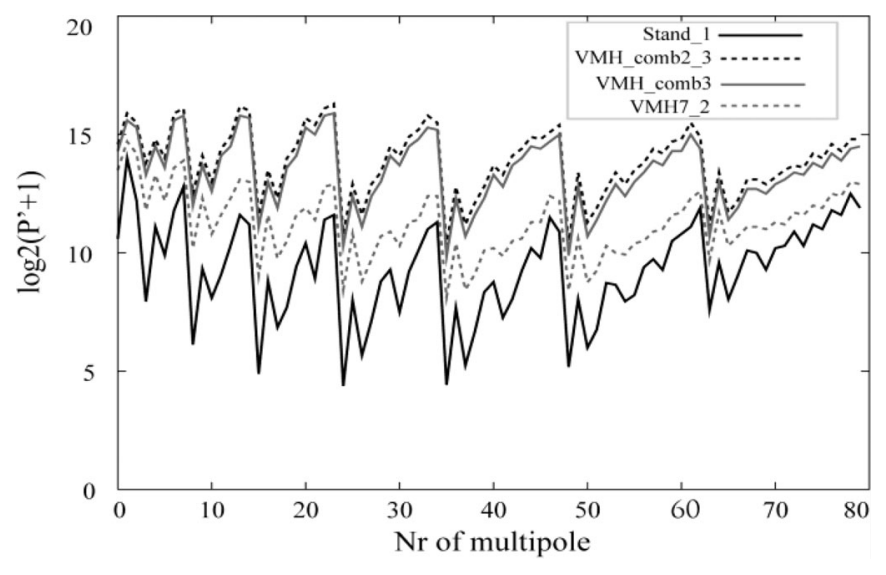

Fig. 5. $\log _{2}$ power SNR of IMMMs computed from the 306-sensor helmet and from VMH using the RDSC model. P'-SNR power of IMMMs.

created by simple displacements (see Table II). There were small differences in total information calculated using IMMM SNR and SLO: Shannon's numbers and PAI (relatively to Stan_1) were somewhat lower using IMMM SNR, than SLO (see Table II).

The comparison of total information calculated using IMMM SNR [12] and using the orthogonalization process on the sensor level [5], [11]. The calculations were based on the RDSC model. The SNR — signal to noise ratio; VMH_comb2_3 - array 22 in Table I. VMH_comb3-array 23 in Table I; VMH7_2array 19 in the Table I. Stand_1-reference head position in standard 306-sensor helmet. PAI-percent of additional information (in Table II, PAI was computed comparing to Stand_1); TI-total information. IMMM-internal multipole moments. SLO—sensor level orthogonalization.

\section{Total Information With Simulated AEFs (see Table I and Fig. 2)}

In simulated AEFs, the single head position with the highest Shannon number was the reference head position (Stand_1). When compared with Stand_1, only one type of VMH (VMH_comb3) had a positive PAI (12.8\%). The same VMH type was the most efficient with RDSC.

\section{E. Total Information With Simulated SEF (see Table I and Fig. 2)}

With simulated SEF, the most informative single head position was Stand_9. When compared with Stand_9, only one type of VMH (VMH4_1) had a positive PAI (1.1\%).

\section{DISCUSSION}

\section{A. Source Model Limitations}

We used two types of simulated neuromagnetic field sources. The first type was RDSC, the source, which is randomly distributed throughout the spherical conductor. This type of source was used in the previous studies in MEG array informatics [5], [11], [12]. The advantage of such a model is its suitability for
Shannon's channel capacity (total information) calculation [see (1) and (2)], which requires independence of signals presented in different channels. RDSC is a useful model, when the sampling ability of different MEG arrays is compared without an assumption regarding specific source location. The independence of channel lead fields can be achieved by the orthogonalization process on the sensor level (see (5)) or by SSS to transform the data into magnetostatic multipole moments. The disadvantage of the RDSC model is that such simulated source is quite far from the real physiological sources. In reality, the source of interest is seldom or ever distributed through the whole brain and always has some degree of spatial smoothness.

The second type of simulated sources were those of evoked fields (AEF and SEF). The AEF sources were simulated by two ECDs located bilaterally, and the SEF source by a single ECD. The advantage of this type of sources is that it is more physiological than RDSC. However, the signal related to the source, which is represented by only one or two dipoles is unavoidably dependant on the multichannel system even after the lead field orthogonalization. Therefore, such source model is not optimal for the Shannon's channel capacity calculation.

Using both types of source models, and taking into account their advantages and disadvantages, enabled us to test $\mathrm{VMH}$ concept from the different angles.

\section{B. Noise Model Limitations}

The calculation of channel capacity (total information) of multichannel device requires that signal and noise are independent. In reality, however, the noise and signal are not independent and the main part of the noise is actually brain background activity ("brain noise"). According to our model, when the head moves closer to sensors, the signal amplitude increases and the noise remains the same, resulting in improved SNR. However, in the reality, the "brain noise" amplitude increases simultaneously with the amplitude increase of the signal of interest. Therefore, the real SNR increase is not as prominent as in our model when head comes closer to the sensors. However, when the head moves away from the sensors, the SNR decrease is less prominent in reality than in our model. This can affect the informatics of different types of the VMH. For example, the RDSC model most probably overestimates the contribution of translations in the $x$-axis from the reference head position and underestimates the contribution of translations in $z$-direction downward or in $y$-direction forward from the reference head position, as in the reference head position, adult sized brain is located close to the posterior and upper walls of the helmet.

\section{Lead Field Orthogonalization}

In order to compute total information, the channels of MEG array should be independent. In reality, however, the lead fields of MEG array are overlapping. This problem can be solved by lead field orthogonalization, which can be achieved either by SLO [5], [11] or by SSS transformation that creates an orthogonal basis of magnetostatic multipole moments. The subset of SSS basis, which corresponds to spherical harmonics inside the MEG sensor sphere, is represented by IMMMs. Both SLO and 
SSS create orthogonal basis suitable for total information calculation. There are, however, some differences. In this study, we demonstrated that values of total information calculated using SLO or SSS bases can be slightly different (see Table II). This can influence to some degree the PAI calculation comparing between VMH and standard array (two lower rows of Table II).

Taking into account the source and noise model limitations and the lead field orthogonalization aspects, we see the role of this study as presenting the general basis of VMH concept. This simulation study cannot quantitatively evaluate the contribution of VMH to the real MEG measurements, which should be investigated further in the studies with real data.

According to the results of our simulations, VMH provided more information than the most informative single head position, in some situations. VMH and single head position were approximately equal in other situations; in many cases, however, VMH provided less information, than the "best" single head position. Several factors influenced the VMH efficiency.

1) Source distribution.

2) Using combined versus simple head displacements.

3) SNR.

\section{Source Distribution}

Using the RDSC model, we demonstrated that MEG recording in different head positions with subsequent VMH construction in some but not all situations can increase the total information extracted from an MEG measurement and increase the IMMM SNR. This can be explained as resulting from addition of channels measuring independent information. Translations correspond to addition of sensor layers and rotations to placement of additional sensors at different angles (see Fig. 1). However, events recorded in different head positions cannot be averaged without movement compensation. Therefore, the noise of the VMH channels is higher than that of the standard helmet channels (when the MEG was recorded in one head position) (see Table I). Nevertheless, the increased number of independent channels can compensate for the less efficient channel noise suppression (see Table I and Fig. 2). The most prominent information gain was associated with VMH types constructed by combined head displacements.

With simulated bilateral AEFs, the majority of VMH types provided less information than the "best" single head position (Stand_1). However, VMH_comb3 (array 23) provided 12.8\% more information than Stand_1. Interestingly the VMH_comb3 was also the most efficient VMH type with RDSC (see Fig. 2).

With simulated unilateral SEFs, VMH did not provide substantial contribution when compared with Stand_9 (the most informative single head position). Only VMH4_1 (array 17) had slightly positive PAI (1.1\%) (see Fig. 2). However, in Stand_9, the source was located close to the sensors in the lateral wall of the helmet, whereas in VMH4_1, the source was close to sensors only part of the time (see Table I). Thus, VMH4_preserved the information content of the simulated MEG recording despite movement of the source away from the sensors. This can be important in the MEG recordings in human subjects (e.g., young children) who cannot keep their head position stable.

\section{E. Using Combined Versus Simple Head Displacements}

The highest PAI with RDSC and the only positive PAI with simulated AEF were achieved by VMH types using combined head displacements (arrays 20-23). Increase in the number of head positions by more than two or three did not necessarily increase the VMH efficiency, because the increase in virtual channel number may not be enough to compensate the increased MEG channel noise. Combined movements were more efficient than simple ones, because they increased the MEG sensor position and orientation variety with minimum decrease in number of averaged events, and therefore, with a minimum increase in MEG channel noise.

\section{F. SNR}

We demonstrated that with higher number of recorded events, and therefore, with higher SNR, the proportional contribution of $\mathrm{VMH}$ increases when compared with the single head position [see Fig. 3 (b) and (c)], whereas the differences in information content between different single head positions decreases [see Fig. 3 (a)]. Based on this, we can expect that application of the data denoising algorithms, such as reported by Taulu et al. [18], can enhance the contribution of the VMH.

\section{G. Practical Application of the VMH Concept}

According to our simulations, VMH can improve the data related to the distributed bilateral source current (RDSC), and possibly also in some situations to the local bilateral sources (AEFs). We, however, did not prove substantial benefit of VMH in the focal unilateral source (SEF). Possibly, the area where $\mathrm{VMH}$ can be most efficiently applied is connectivity studies of the complex bilateral brain networks with stable spatiotemporal hierarchy.

The sources located close to the head surface have higher spatial frequencies than deep sources. Because the signal amplitude nonlinearly depends on the source-to-sensor distance, the higher spatial frequencies (signals related to more superficial sources) are more influenced by head displacements. Therefore, we expect that VMH will be more informative with more superficial sources.

The VMH concept demonstrates how the information may increase with different head positions. However, whether or not the same information increment persists after transforming the measurements into a single position by a suitable movement compensation algorithm based on minimum norm estimate [19] or on SSS [14], needs to be investigated in further studies.

In order to construct a VMH, the same brain activity should be recorded in different head positions. This is relatively easy with evoked responses, because the nature of the stimulus and its timing are controlled by operator. With interictal epileptic spikes, the situation is more complex. According to some studies, it is possible to classify and average epileptic spikes [20], but the same type of spikes recorded in different head positions have different amplitude distribution pattern across the MEG channels. To classify spikes, the MEG trace can be recalculated into one head position using movement compensation [6], [13], or the MEG data can be transformed into source montage 
[21]. Alternatively, spikes can be classified using simultaneously recorded EEG, because the amplitude distribution in EEG does not depend on head position. Due to spatial instability, however, the application of VMH to the epileptic spike analysis is a controversial issue.

The observed increase of IMMM SNR proves that with VMH the measured magnetic field can be represented by numerically more stable vector space basis. This opens the perspective for more robust source estimation, which should be evaluated by further studies including real subject recordings.

Head movements inside MEG helmet are traditionally considered as an obstacle. On the contrary, our study shows that head movements (or recording in different head positions) in some situations can actually improve the quality of MEG data. Not all movements are beneficial for MEG data. In our simulations, several types of $\mathrm{VMH}$ actually reduced the total information of MEG measurements. With large displacements of the head, the distance between source and sensors can increase, thereby, worsening SNR.

There are some specific issues, which should be taken into account using a VMH approach. For example, experiments including visual stimulus, can be affected by changing head position. Therefore, visual stimulus parameters such as stimulus position and size probably have to be updated according to different head position. Another example relates to the fact that the MEG helmet is not complete sphere, and therefore, some sources are located close to the sensor array margin. In such cases, relatively small head displacements can remove those "marginal" sources out of the sensor array, preventing adequate field sampling. This can be true for anterior temporal and basal frontal sources. On the other hand, however, VMH can optimize evaluation of "marginal" sources. For example, when left and right anterior temporal lobe are activated simultaneously, head rotation to the left can improve left anterior temporal source field sampling and vice versa. Subsequent data interpolation can reconstruct the complete picture of bilateral anterior temporal activity dynamics in space and in time.

The important question is how to test the VMH concept using real data. Investigation of this question is not trivial. Because the real source and real noise are not distributed randomly, Shannon's channel capacity computing can be not an optimal way for the VMH concept evaluation using real data. Probably, the best way is the source localization assessment. However, it is also not trivial to compare between different source solutions. Because MEG source localization is an ill-posed problem, the criteria of the solution correctness are not always robust. The interesting possibility is to compare the source solutions not according to correctness, rather according to stability, changing parameters of source localization process (e.g., using L1 and L2 norms). The more informative neuromagnetic field sampling is expected to result in more stable source solutions. This, however, should be tested in the further studies.

\section{CONCLUSION}

MEG recording in different head positions with subsequent virtual helmet construction can in some circumstances increase the total information and the vector space basis stability without an increase in recording time. More distributed bilateral brain activity, combined head movements and higher SNR can be associated with higher VMH efficiency. We did not prove that VMH is more efficient than single head position in the situations, when the source current is restricted to a small brain area and can be easily explained by one equivalent current dipole. These results justify further studies of VMH in basic neuroscience and clinical practice.

\section{ACKNOWLEDGMENT}

The authors would like to thank Prof. J. Sarvas, Prof. M. Hämäläinen, Prof. R. Ilmoniemi, Prof. M. Scherg, and Prof. T. Stroganova for important discussion and valuable suggestions and Mrs. M. Ovadia for assistance in graphical work.

\section{REFERENCES}

[1] J. Nurminen, S. Taulu, and Y. Okada, "Improving the performance of the signal space separation method by comprehensive spatial sampling," Phys. Med. Biol., vol. 55, no. 5, pp. 1491-1503, 2010.

[2] J. Nurminen, S. Taulu, J. Nenonen, L. Helle, J. Simola, and A. Ahonen, "Improving MEG performance with additional tangential sensors," IEEE Trans. Biomed. Eng., vol. 60, no. 9, pp. 2559-2566, Sep. 2013.

[3] J. S. Vrba, E. Robinson, and J. McCubbin, "How many channels are needed for MEG?" Neurol. Clin. Neurophysiol., vol. 2004, pp. 99-99, 2004.

[4] C. E. Shannon, "Communication in the presence of noise," Proc IRE, vol. 37, no. 1, pp. 10-21, Jan. 1949.

[5] P. Kemppainen and R. J. Ilmoniemi, "Channel capacity of multichannel magnetometers," in Advances in Biomagnetism, S. Williamson, M. Hoke, G. Stroink, and M. Kotani, Eds. New York, NY, USA: Plenum Press, 1989 , pp. 635-638.

[6] S. Taulu and M. Kajola, "Presentation of electromagnetic multichannel data: The signal space separation method," J. Appl. Phys., vol. 97, pp. 124905-1-124905-10, 2005.

[7] D. S. Barth, W. Sutherling, J. Engel Jr, and J. Beatty, "Neuromagnetic localization of epileptiform spike activity in the human brain," Science, vol. 218, pp. 891-894, 1982.

[8] W. W. Sutherling, P. H. Crandall, J. Engel, T. M. Darcey, L. D. Cahan, and D. S. Barth, "The magnetic field of complex partial seizures agrees with intracranial localizations," Ann. Neurol., vol. 21, no. 6, pp. 548-558, 1987.

[9] J. E. T. Knuutila, A. I. Ahonen, M. S. Hämäläinen, M. J. Kajola, P. P. Laine, O. V. Lounasmaa, L. T. Parkkonen, J. T. Simola, and C. D. Tesche, "A 122-channel whole-cortex SQUID system for measuring the brain's magnetic fields," IEEE Trans. Magn., vol. 29, no. 69, pp. 3315-20, Nov. 1993.

[10] M. Medvedovsky, R. Paetau, J. P. Mäkelä, A. Ahonen, J. Nenonen, J. Nurminen, J. Simola, and S. Taulu, "Virtual MEG helmet," presented at the ISACM meeting, Sapporo, Japan, Aug. 28-30, 2013.

[11] J. Nenonen, M. Kajola, J. Simola, and A. Ahonen, "Total information of multichannel MEG sensor arrays," in Proc. 14th Int. Conf. Biomagn., 2004, pp. 630-638.

[12] J. Nenonen, S. Taulu, M. Kajola, and A. Ahonen, "Total information extracted from MEG measurements," Int. Congr. Ser., vol. 1300, pp. 245$248,2007$.

[13] S. Taulu and J. Simola, "Spatiotemporal signal space separation method for rejecting nearby interference in MEG measurements," Phys. Med. Biol., vol. 51, pp. 1759-1768, 2006

[14] S. Taulu, J. Simola, and M. Kajola, "Applications of the signal space separation method," IEEE Trans. Signal Process., vol. 53, no. 9, pp. 33593372 , Sep. 2005

[15] J. Nenonen, J. Nurminen, D. Kicic, R. Bikmullina, P. Lioumis, V. Jousmäki, S. Taulu, L. Parkkonen, M. Putaala, and S. Kähkönen, "Validation of head movement correction and spatiotemporal signal space separation in magnetoencephalography," Clin. Neurophysiol., vol. 123, pp. 2180-2191, 2012.

[16] J. Nenonen, L. Parkkonen, L. Helle, S. Taulu, and A. Ahonen, "Repeatability of AEF and SEF from static and moving head positions," in 17th 
International Conference on Biomagnetism Advances in BiomagnetismBiomag 2010. Berlin, Germany: Springer, Jan. 2010, pp. 306-309.

[17] D. T Wehner, M. S. Hämäläinen, M. Mody, and S. P. Ahlfors, "Head movements of children in MEG: Quantification, effects on source estimation, and compensation," Neuroimage, vol. 40, no. 2, pp. 541-550.

[18] S. Taulu, J. Simola, M. Kajola, L. Helle, A. Ahonen, and J. Sarvas, "Suppression of uncorrelated sensor noise and artifacts in multichannel MEG data," presented at the 18th Int. Conf. Biomagnetism, Paris, France, 2012.

[19] K. Uutela, S. Taulu, and M. Hämäläinen, "Detecting and correcting for head movements in neuromagnetic measurements," Neuroimage, vol. 14, no. 6 , pp. 1424-1431, 2001
[20] T. Bast, O. Oezcan, S. Rona, C. Strippich, A. Seitz, A. Rupp, S. Fauser, J. Zenter, D. Rating, and M. Scherg, "EEG and MEG source analysis of single and averaged interictal spikes reveals intrinsic epileptogenicity in focal cortical dysplasia," Epilepsia, vol. 45, pp. 621-631, 2004.

[21] K. Hoechstetter, H. Bornfleth, D. Wecksler, N. Ille, P. Berg, and M. Scherg, "BESA source coherence: A new method to study cortical oscillatory coupling," Brain Topogr., vol. 16, no. 4, pp. 233-238, 2004.

Authors' photographs and biographies not available at the time of publication. 